\title{
QUICKPIC: A PARALLELIZED QUASI-STATIC PIC CODE FOR MODELING PLASMA WAKEFIELD ACCELERATION
}

\author{
Chengkun Huang, Viktor Decyk, Shuoqin Wang, Evan S. Dodd, Chuang Ren, Warren B. Mori \\ University of California Los Angeles, Los Angeles, CA 90095 \\ Tom Katsouleas \\ University of Southern California, Los Angeles, CA 90089 \\ Tom Antonsen Jr. \\ University of Maryland, College Park, MD 20742
}

\begin{abstract}
There has been much recent interest in plasma wakefield acceleration. This is partly due to the possibility of using it as an energy doubler, i.e., an afterburner, stage at the end of an existing linear collider such as SLC. The process in this scheme is highly nonlinear and therefore particle models are required to study it. Furthermore, the key physics involves fully three-dimensional effects. Unfortunately, even on the largest computers it is still not possible to model a full energy doubler stage using existing codes such as OSIRIS [1]. Fortunately however, for these cases the drive beam evolves on a much longer time scale than the plasma frequency. In these cases the beam appears static or frozen for long periods of time. Under these conditions one can make the quasi-static or frozen field approximation. We have recently developed a skeleton version of a parallelized quasi-static PIC code for modeling particle beam drivers. This code combines all the best features from WAKE [2] and the work of D. H. Whittum [3]; and it is fully parallelized. We describe the basic equations and the algorithm. We will also present preliminary results, which include benchmarking it against our fully explicit code OSIRIS.
\end{abstract}

\section{INTRODUCTION}

When a highly relativistic electron beam is sent through the background plasma, plasma electrons are expelled by the drive beam, while ions can be considered motionless due to the fact that they are much heavier than electrons. If the background plasma density is lower than the beam density ("underdense"), all the electrons surrounding the drive beam are expelled and an ion channel is formed. Inside the ion channel, the focusing field is linear. The longitudinal wakefield can provide acceleration to the electrons at the tail of the drive beam.

The focusing field witnessed by the electrons in the drive beam causes them to oscillate in the transverse direction. This leads to the beam envelope undergoing oscillations at the betatron frequency $\omega_{\beta}=\omega_{p} / \sqrt{2 \gamma_{b}}$ where $\omega_{\mathrm{p}}$ is the plasma frequency. When the drive beam is highly relativistic, it evolves on time scales much longer than the plasma frequency. Thus, the quasi-static or frozen field approximation can be used to simplify the analysis.

Theoretical analysis [4] and computer simulation [5] using a reduced set of the quasi-static approximation have already been carried out. These results predict that the hosing instability of the drive beam will occur under the E157 experiment parameters [5] and will be very severe under possible Afterburner parameters.

But recent results from E157 experiment and computer simulation using 3D fully electromagnetic code OSIRIS [6] showed less hosing than the analysis and simulation based on the simplified theory. For the afterburner parameters, this discrepancy became even clearer, suggesting that more physics needs to be included in our analysis.

Such consideration motivated the development of QuickPIC, a parallelized quasi-static PIC code for modeling plasma wakefield acceleration. QuickPIC is aimed to solve the full quasi-static equations. We hope that it will then reproduce the results of the fully explicit, electromagnetic PIC code OSIRIS. Currently a version of QuickPIC with relativistic mass effects, longitudinal motion of plasma electrons and parallel plasma current corrections has been implemented. More details about the code are presented in Sec. 2, and some preliminary results are given in Sec. 3.

\section{QUASI-STATIC PIC CODE: QUICKPIC}

We started from the Maxwell equations in Lorentz guage First, we change variables to $s$ and $\xi$, where $s=z, \xi=z-c t$. Then, quasi-static approximation is taken, i.e., $\partial_{s} \approx 0$. We neglect the longitudinal motion of beam electrons and plasma electrons; also assume that the total current can be approximated by the dominant beam current,

$\mathbf{j}=\mathbf{j}_{b}+\mathbf{j}_{e} \approx \mathbf{j}_{b}=c \rho_{b} \hat{z}$

Thus a set of basic quasi-static equations for QuickPIC can be written as,

Field equations:

$$
\nabla_{\perp}^{2} \phi=-4 \pi\left(\rho_{b}+\rho_{e}+\rho_{i o n}\right)
$$$$
\nabla_{\perp}^{2} \Psi=-4 \pi\left(\rho_{e}+\rho_{i o n}\right)
$$ 
Equations of motion:

$$
\begin{aligned}
& \frac{d \mathbf{V}_{e \perp}}{d \xi}=q_{e} \nabla_{\perp} \phi \\
& \frac{d \mathbf{X}_{e \perp}}{d \xi}=\mathbf{V}_{e \perp} \\
& \frac{d \mathbf{P}_{b \perp}}{d s}=q_{b} \nabla_{\perp} \Psi \\
& \frac{d \mathbf{X}_{b \perp}}{d s}=\mathbf{P}_{b \perp} / \gamma_{b}
\end{aligned}
$$

In (2), $\Psi=\phi-A_{/ /}$, where $\phi$ is the electric potential and $\mathbf{A}=A_{/ /} \hat{z}$ is the vector potential of magnetic field. The subscripts e, ion, and $\mathrm{b}$ in Eq. (1)-(6) denote plasma electron, ion and beam respectively.

The transverse evolution is completely decoupled from the longitudinal evolution in Eq. (1)-(6). In QuickPIC, Eq. (1) and (2) are solved in 2D Fourier space with periodic boundary condition by a parallel Poisson solver. The velocity and position of plasma electrons are updated every 2D time step, $\Delta \xi$, which needs to be chosen to resolve the plasma frequency. While the momentum and position of the beam electrons are updated every $3 \mathrm{D}$ time step, $\Delta s$, which needs only resolve the betatron frequency or the hosing growth. Such an algorithm can achieve a speed up of about 2 orders of magnitude over a fully explicit code such as OSIRIS when modeling an E157 type of experiment. The above algorithm is identical to that of D. H. Whittum [3].

As we will show shortly, by comparing results using this algorithm to those from OSIRIS, we realize that the above approximations are too severe. To rectify this, we have begun to implement the full set of quasi-static equations into QuickPIC. We started by making several modifications to the basic equations while keeping the fundamental code structure unchanged.

The first modification is to include relativistic mass effects of the plasma electrons. This is done by simply upgrading to a relativistic plasma electron pusher.

The longitudinal motion of plasma electrons can also be introduced into the structure of QuickPIC by the following correction to the plasma charge density [3],

$$
\rho_{e}=\frac{1}{V} \sum_{i} \frac{q_{e i}}{1-V_{e / / i} / c}
$$

accordingly, equations (1) and (3) become,

$$
\begin{aligned}
& \nabla_{\perp}^{2} \phi=-4 \pi\left(\rho_{b}+\sum_{i} \frac{q_{e i}}{1-V_{e / / i} / c}+\rho_{\text {ion }}\right) \\
& \frac{d \mathbf{P}_{e \perp i}}{d \xi}=\frac{q_{e i}}{1-V_{e / / i} / c} \nabla_{\perp} \phi
\end{aligned}
$$

The parallel current of plasma can also be included in a similar way,

$$
\mathbf{j}_{e / /}=\frac{1}{V} \sum_{i} \frac{q_{e i} V_{e / / i}}{1-V_{e / / i} / c} \hat{z}
$$

thus,

$$
\nabla_{\perp}^{2} \Psi=-4 \pi\left(\rho_{e 0}+\rho_{i o n}\right)
$$

where,

$$
\rho_{e 0}=\frac{1}{V} \sum_{i} q_{e i}
$$

Last, the transverse currents due to the plasma and beam need to be taken into account. The transverse current, $\mathbf{j}_{\perp}$, generates a transverse vector potential,

$$
\nabla_{\perp}^{2} \mathbf{A}_{\perp}=-\frac{4 \pi}{c} \mathbf{j}_{\perp}
$$

which produces extra magnetic fields. Therefore, a pusher using the full Lorentz force is required.

Currently, QuickPIC includes relativistic mass effects, the longitudinal motion of plasma electrons, parallel plasma current. The code is also parallelized using MPI. Next, we plan on including the transverse current corrections and properly time centering the code. These are needed since with the first three corrections, QuickPIC results still differences with the results from OSIRIS.

\section{PRELIMINARY RESULTS}

QuickPIC has been used to simulate the electron hosing instability and longitudinal wakefield acceleration based on E157 / afterburner beam and plasma parameters. For the simulation of E157 experiment, plasma density was $2.1 \times 10^{14} \mathrm{~cm}^{-3}$. Beam charge was $1.8 \times 10^{10}$ electrons, charge density had a Gaussian distribution with $\sigma_{\mathrm{r}}=0.07 \mathrm{~mm}$ and $\sigma_{\xi}=0.63 \mathrm{~mm}$. The energy of beam was $30 \mathrm{Gev}(\gamma=60,000)$. The dimentions of simulation was $8 \times 8 \times 17.6 \mathrm{c} / \omega_{\mathrm{p}} \quad(2.9 \times 2.9 \times 6.4 \mathrm{~mm}), \quad$ divided into $128 \times 128 \times 256$ cells. $128 \times 128 \times 320$ particles were used for the beam and $512 \times 512$ particles for the plasma. For the afterburner run, plasma density was $2.1 \times 10^{16} \mathrm{~cm}^{-3}$, spot size $\sigma_{\mathrm{r}}=0.007 \mathrm{~mm}$ and $\sigma_{\xi}=0.063 \mathrm{~mm}$ (the beam density normalized to the plasma density is ten times higher.) The dimensions were $24 \times 24 \times 16 \mathrm{c} / \omega_{\mathrm{p}}(0.88 \times 0.88 \times 0.59 \mathrm{~mm})$, with $256 \times 256 \times 256$ cells in each dimension. Other parameters were the same as E157 simulation. Preliminary results show that the magnitudes of the focusing fields are consistent with that from OSIRIS. But at present the code does not fully reproduce the accelerating fields from OSIRIS (Fig 1).

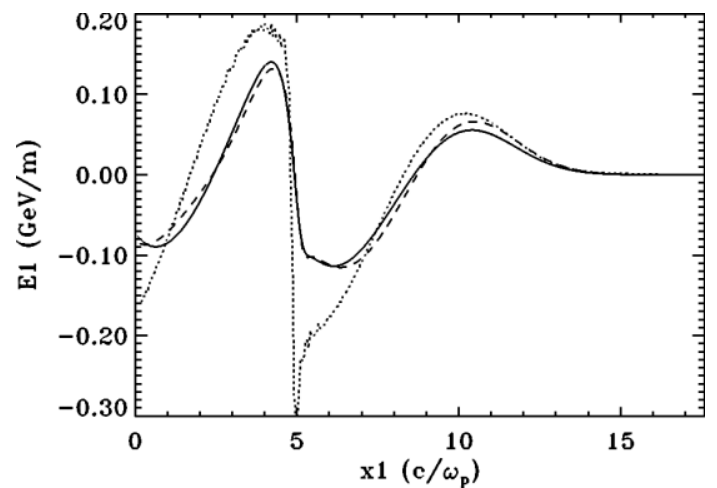

Fig.1: Longitudinal accelerating field (E157 parameters) from QuickPIC(solid, with corrections; thick dash, without corrections) and OSIRIS (thin dash) 


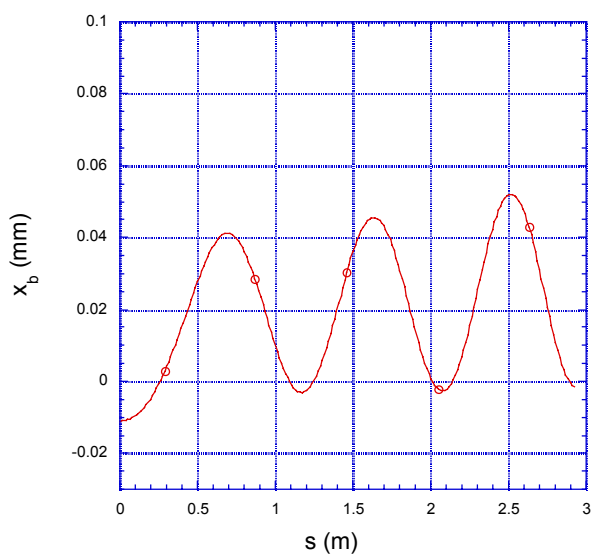

Fig.2: beam centroid oscillation (E157 parameters, with corrections).

Sample hosing results are shown in figure 2 and 3. Fig.2 shows the growth of centroid oscillation from a QuickPIC E157 run. All three of the existing corrections were turned on in this run. The measured growth rate is actually smaller than the theoretical prediction and a little larger than the OSIRIS result. In fig. 3, the same graph from an afterburner run is shown. For this case, the basic quasi-static equations were solved with no additional corrections.

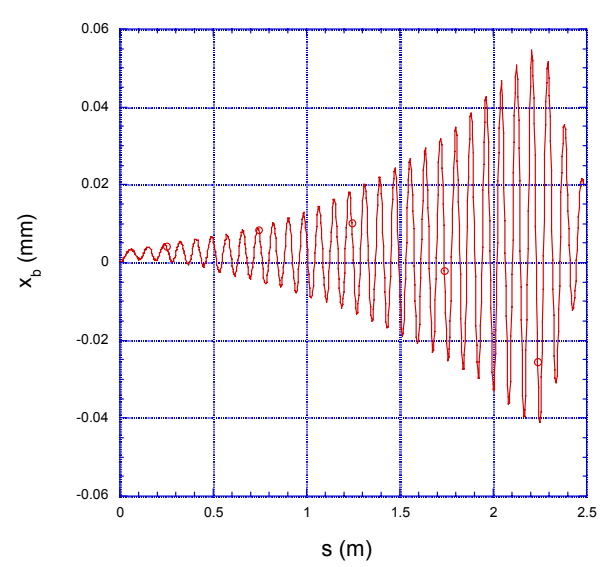

Fig.3: beam centroid oscillation (Afterburner parameters, without corrections)

\section{CONCLUSION}

A new parallelized quasi-static code called QuickPIC was described. Preliminary results from this code were presented and the results for hosing lie between those of simplified theory and those from the fully explicit, electromagnetic code OSIRIS. In the future, we plan to add the full set of quasi-static equations to QuickPIC in the hope that it will then reproduce results from OSIRIS and experiments.

This work was supported by DOE under contract number DE-FG03-92ER40727 and by NSF grants DMS9722121 and ECS-9617089.

\section{REFERENCE}

[1] R. G. Hemker et. al., Proc. 1999 Part. Accel. Conf., (1999)

[2] P. Mora and T. M. Antonsen, Phys. Plasmas 4, 217 (1997)

[3] D. H. Whittum, Phys. Plasmas, 4, 1154 (1997)

[4] D. H. Whittum et. al., Phys. Rev. Lett., 67, 991 (1991)

[5] A. A. Geraci and D. H. Whittum, Phys. Plasmas, 7, $3431(2000)$.

[6] E.S. Dodd, et. al. submitted to Phys. Rev. Lett. 